\title{
Principais intervenções de enfermagem no apoio a cuidadores familiares: uma revisão integrativa
}

RESUMO | Objetivo: Identificar as principais intervenções de enfermagem no apoio a cuidadores familiares. Métodos: Trata-se de uma revisão integrativa, realizada nas bases de dados: SciELO, Scopus, NIH-PUBMED e Web of Science. Foram utilizados os seguintes descritores extraídos do Medical Subject Headings (MeSH): "care", "home care", "family caregivers" e "nursing". Após o processo de busca, foram recuperados 844 artigos, de modo que apenas 13 atenderam aos critérios de inclusão, compondo a amostra final. Resultados: Em relação ao desenho metodológico a maior parte utilizou o ensaio clínico randomizado (n: 09), com predominância do nível de evidência II. Os estudos apresentaram componentes de intervenção, de modo que alguns apresentaram intervenções mistas: suporte prático ao cuidar (n: 09); avaliação de necessidades (n: 05) e; psicoeducação (n: 04). Conclusão: Verificou-se que as intervenções observadas tiveram efeitos positivos entre os cuidadores familiares, sugerindo que a enfermagem tem função importante no apoio a estes indivíduos.

Palavras-chaves: Autocuidado; Assistência Domiciliar; Cuidadores; Enfermagem.

\begin{abstract}
I Objective: Identify the main nursing interventions to support family caregivers. Methods: This is an integrative review, carried out on the databases: SciELO, Scopus, NIH-PUBMED and Web of Science. The following descriptors from the Medical Subject Headings (MeSH) were used: "care", "home care", "family caregivers" and "nursing". After the search process, 844 articles were retrieved, so that only 13 met the inclusion criteria, making up the final sample. Results: Regarding the methodological design, most used the randomized clinical trial (n: 09), with a predominance of evidence level II. The studies presented intervention components, so that some presented mixed interventions: practical support when caring (n: 09); needs assessment (n: 05) and; psychoeducation (n: 04). Conclusion: It was found that the observed interventions had positive effects among family caregivers, suggesting that nursing has an important role in supporting these individuals.
\end{abstract}

Keywords: Self Care; Home Nursing; Caregivers; Nursing.

RESUMEN | Objetivo: Identificar las principales intervenciones de enfermería para apoyar a los cuidadores familiares. Métodos: Se trata de una revisión integradora, realizada sobre las bases de datos: SciELO, Scopus, NIH-PUBMED y Web of Science. Se utilizaron los siguientes descriptores de Medical Subject Headings (MeSH): "cuidados", "cuidados domiciliarios", "cuidadores familiares" y "enfermería". Tras el proceso de búsqueda se recuperaron 844 artículos, por lo que solo 13 cumplieron los criterios de inclusión, conformando la muestra final. Resultados: En cuanto al diseño metodológico, la mayoría utilizó el ensayo clínico aleatorizado (n: 09), con predominio del nivel de evidencia II. Los estudios presentaron componentes de intervención, por lo que algunos presentaron intervenciones mixtas: apoyo práctico en el cuidado (n: 09); evaluación de necesidades ( $\mathrm{n}$ : 05) y; psicoeducación (n: 04). Conclusión: Se encontró que las intervenciones observadas tuvieron efectos positivos entre los cuidadores familiares, lo que sugiere que la enfermería tiene un papel importante en el apoyo a estos individuos.

Palabras claves: Autocuidado; Atención Domiciliaria de Salud; Cuidadores; Enfermería.

\section{Ana Lúcia Barros Silva}

Enfermeira graduada pela Universidade

Católica de Pernambuco.

ORCID: 0000-0003-2140-0815

\section{José William Araújo do Nascimento}

Enfermeiro (Universidade Católica de Pernambuco), Mestrando em Ciência da Computação (Universidade Federal de Pernambuco), Membro do Grupo de Pesquisa em Estomaterapia (Universidade de Pernambuco) e Sócio da Sociedade Brasileira de Anatomia (SBA).

ORCID: 0000-0002-1844-1117

\section{Fernando Ramos Gonçalves}

Enfermeiro (Universidade Federal de Pernambuco), Residência em Medicina Preventiva e Social (FIOCRUZ), Mestre em Saúde Pública (CENTRO DE PESQUISA AGGEU MAGALHÃES / FIOCRUZ), Doutorando em Ciências das Linguagens (Universidade (atólica de Pernambuco), Docente da FENSG-UPE e Universidade Católica de Pernambuco (Enfermagem e Medicina), Enfermeiro intensivista da Unidade de Suporte Avançado em Neurocirurgia-USAN/ Hospital da Restauração e Preceptor da Residência de Enfermagem em Terapia Intensiva e Residência de Enfermagem em Neurologia/Neurocirurgia.

ORCID: 0000-0003-2692-9769
INTRODUÇÃO


iante da situação atual de envelhecimento demográfico, as últimas oito décadas acrescentaram mais de trinta anos na expectativa de vida da população(1), que resultaram no aumento da prevalência de doenças crônicas não transmissíveis (DCNT).

$\mathrm{O}$ exposto traz à luz circunstâncias que vêm se tornando comum no cotidiano de muitas famílias: indivíduos portadores de enfermidades que se encontram limitados para realização das atividades de vida diária (AVDs), que podem apenas contar com a ajuda de parentes para conseguir realizá-las ${ }^{(2)}$.

Quando surge um paciente com doenças incapacitantes ou restritivas 
de realização de atividades básicas do dia-a-dia, surge também um cuidador e grande parte destes, vão se originar do núcleo e âmbito familiar ${ }^{(3)}$. A ocupação de cuidador é então definida como aquela que zela e ampara alguém em suas funções necessárias para a saúde e bem-estar ${ }^{(4)}$, proporcionando maior qualidade de vida à pessoa que dele esteja necessitando por estar acamada ou com limitações físicas ${ }^{(5)}$.

No Brasil, a política de atenção domiciliar determina que os profissionais de saúde desta rede de atenção, sejam os responsáveis por treinar os cuidadores familiares e envolvê-los no processo de cuidar $^{(6)}$. A ausência de treinamento e informações geram ansiedade e déficit no autocuidado dos acompanhantes, que ficam mais expostos a desequilíbrios na sua saúde física e emocional ${ }^{(7)}$.

Embora as políticas públicas de saúde brasileiras norteiem a estruturação de uma rede de atenção à saúde, elas apresentam vulnerabilidade e serviços de saúde ineficientes no envolvimento dos cuidadores. A enfermagem, que foca em fornecer suporte para pacientes, precisa então, reconhecer a necessidade de estabelecer programas específicos para identificar e suprir as necessidades dos cuidadores-familiares ${ }^{(3,8)}$.

No âmbito domiciliar, o enfermeiro se caracteriza por ser o profissional dotado de conhecimento e habilidades que objetivam a educação profissional dos cuidadores familiares, além de desempenhar uma função primordial na orientação dos pacientes que recebem a assistência destes profissionais bem como de seus familiares ${ }^{(8,9)}$.

De modo que a maior parte das pesquisas disponíveis na literatura, referente aos cuidadores familiares, sejam relacionadas às características familiares e sociais dos mesmos, além da sobrecarga de trabalho e qualidade de vida, poucos estudos existem que apresentem intervenções de enfermagem que atendam às necessidades destes cuidadores ${ }^{(8)}$.
Nessa perspectiva, o presente estudo objetivou identificar na literatura científica as principais intervenções de enfermagem no apoio a cuidadores familiares, e suas dificuldades quando auxiliando membros da família nas suas AVDs.

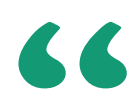

No âmbito domiciliar, o enfermeiro se caracteriza por ser o profissional dotado de conhecimento e habilidades que objetivam a educação profissional dos cuidadores familiares, além de desempenhar uma função primordial na orientação dos pacientes que recebem a assistência destes profissionais bem como de seus familiares.

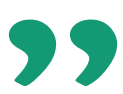

\section{MÉTODOS}

Trata-se de um estudo de revisão in- tegrativa, que se caracteriza pela análise ampla da literatura, com o objetivo de obter um completo entendimento de um determinado tema ${ }^{(10)}$. O percurso metodológico compreendeu cinco fases distintas: identificação do tema ou questionamento da revisão integrativa; busca na literatura e categorização das publicações; agrupamento e análise dos estudos incluídos; interpretação dos resultados e; síntese do conhecimento da amostra final ${ }^{(11)}$.

Buscou-se, responder a seguinte questão norteadora: Quais as principais intervenções de enfermagem à cuidadores familiares? Partindo desta questão, foi realizada a busca por três pesquisadores independentes, nas bases de dados: Scientific Eletronic Libray Online (SciELO), SCOPUS, Nacional institute of Medicine (NIH-PUBMED) e Web of Science.

Para a busca nas bases de dados, foram combinados os seguintes descritores por meio de operadores boleanos: "family caregivers" AND "care" AND "nursing interventions". Os descritores classificados foram retirados do Medical Subject Headings (MeSH).

Para a realização das buscas, foram adotados os seguintes critérios de inclusão: artigos completos em português, inglês ou espanhol e que descrevessem claramente o papel do enfermeiro em intervenções aos cuidadores familiares. Foram excluídas publicações com temática não relevante ao objetivo desta pesquisa, aqueles metodologias não apresentassem clareza na descrição das intervenções, estudos cuja intervenções eram exclusivamente de cunho psicológico dos cuidadores familiares, pesquisas com intervenções voltadas ao paciente domiciliar, estudos de revisão e duplicatas. Não foi estabelecido limite temporal nas buscas.

Os artigos encontrados nas bases de dados descritas foram então postos a uma leitura exploratória, a fim de identifica-los e, consequentemente, extrair as variáveis. Na sequência, foi utilizado no Software Microsoft Excel 2013, um instrumento de buscas ${ }^{(12)}$ adaptado para 
este estudo, para tabulação das seguintes variáveis: título do artigo, autores, periódico, ano de publicação e país de origem do estudo; qualis dos periódicos conforme dados da Coordenação de Aperfeiçoamento de Pessoal de Nível Superior (Capes) na área de enfermagem; base de dados; tipo do estudo e nível de evidência; patologia ou situação clínica envolvida; intervenções de enfermagem e; resultados das intervenções.

As buscas foram desenvolvidas de março a maio de 2020. Foram encontrados 884 artigos, dos quais 411 nas Web of Science, 260 na PubMed, 125 na Scopus e 88 na Scielo. Após a leitura dos títulos, 95 artigos foram excluídos por serem duplicatas. Após a leitura dos resumos, 708 artigos foram excluídos por não serem de abordagem relevante ao estudo e por não atenderem a outros critérios de elegibilidade; 61 publicações foram então pré-selecionadas para leitura na íntegra; 24 foram excluídas por apresentarem apenas intervenções de cunho psicológico, 20 por apresentarem intervenções voltadas ao paciente domiciliar e 04 por não apresentarem com clareza a intervenção proposta. Desta forma, 13 artigos compuseram a amostra final do estudo, conforme se observa na Figura 1.

Para definição dos níveis de evidência, foi utilizado a classificação proposta por Stillwell et $\mathrm{al}^{(13)}$. Destaca-se que esta pesquisa, por ser um estudo de revisão, respeitou as conformações éticas mediante a Lei de Direitos Autorais no 9.610 de 19 de Fevereiro de $1998^{(14)}$, de modo que todos os autores das publicações analisadas foram referenciados apropriadamente.

\section{RESULTADOS}

Do total de 13 artigos analisados, foi observado maior frequência de publicação no ano de 2015 (n: 04) e maior produção da Austrália (n: 05). O periódico mais frequentemente utilizado para publicação dos estudos foi o PsychoOn-

\section{Figura 1: Fluxograma do processo de seleção do estudo, Recife, PE, Brasil, 2020.}



Fonte: Dados da pesquisa. cology (n: 03), enquanto que em relação aos qualis dos periódicos da amostra, 05 são classificados como A1 na área de enfermagem pela Capes. Quanto às bases de dados, cinco publicações estavam indexadas na Pubmed, seguido pela Web of Science (n: 04), Scopus (n:03), e Scielo (n: 01). Em relação ao desenho metodológico dos artigos analisados, verificou-se que a maior parte utilizou o ensaio clínico randomizado (ECR) (n: 09), de modo que o nível de evidência mais comum tenha sido um foi o II.

O Quadro 1 elucida as principais intervenções de enfermagem encontradas nos artigos analisados. Verifica-se que maior parte dos estudos propôs intervenções à cuidadores familiares com pacientes oncológicos em cuidado paliativo (n: 09), enquanto que outros em pacientes com sequelas de acidente vascular encefálico (n: 05). Os estudos apresentaram componentes de intervenção, de modo que alguns apresentaram intervenções mistas: suporte prático ao cuidar (n: 09); avaliação de necessidades (n: 05) e; psicoeducação (n: 04).

Os resultados identificados após as intervenções com os cuidadores familiares mostraram que as ações realizadas por enfermeiros provocaram melhorias no aspecto prático do cuidar, como na solução de problemas e competências no autocuidado, e também nas habilidades de enfrentamento das situações adversas, como se lidar com o luto em pacientes de cuidados paliativos. Alguns aspectos psicológicos também foram avaliados como taxas de menor depressão e sofrimento em detrimento dos grupos controles avaliados pelos estudos.

Quadro 1: Caracterização das intervenções de enfermagem a cuidadores familiares, Recife/PE, Brasil, 2020.

\begin{tabular}{|l|c|c|}
\hline Situação clínica & Intervenção de enfermagem & Resultados pós-intervenção \\
\hline *AVE(15) & $\begin{array}{c}\text { Visita domiciliar com treinamento de habilidades para a } \\
\text { solução de problemas, seguido de contatos telefônicos } \\
\text { semanais. (Suporte prático ao cuidar) }\end{array}$ & $\begin{array}{c}\text { Melhores habilidades de solução de problemas; maior } \\
\text { preparo do cuidador; menos depressão; e melhoria nas me- } \\
\text { didas de vitalidade, funcionamento social e saúde mental. }\end{array}$ \\
\hline Oncologia ${ }^{(16)}$ & $\begin{array}{c}\text { Duas visitas domiciliares e uma ligação telefônica de } \\
\text { acompanhamento entre as duas visitas. (Suporte prático ao } \\
\text { cuidar) }\end{array}$ & $\begin{array}{c}\text { Experiência mais positiva frente aos cuidados paliativos no } \\
\text { grupo de intervenção. }\end{array}$ \\
\hline
\end{tabular}




\begin{tabular}{|c|c|c|}
\hline Oncologia ${ }^{(17)}$ & $\begin{array}{l}\text { Grupo psicoeducacional com três sessões em um período } \\
\text { de } 3 \text { semanas. (Psicoeducação) }\end{array}$ & $\begin{array}{c}\text { Efeitos positivos na preparação para o papel, competência } \\
\text { e recompensas do cuidar. }\end{array}$ \\
\hline Oncologia ${ }^{(18)}$ & $\begin{array}{c}\text { Consistiu na preparação e avaliação dos cuidadores e na } \\
\text { elaboração de um plano de assistência. (Suporte prático ao } \\
\text { cuidar e avaliação de necessidades) }\end{array}$ & $\begin{array}{c}\text { Melhorias nos níveis de preparação e competência dos } \\
\text { cuidadores. }\end{array}$ \\
\hline${ }^{*} \mathrm{AVE} \mathrm{E}^{(19)}$ & $\begin{array}{l}0 \text { grupo randomizado recebeu uma média de 36,7 h de } \\
\text { psicoeducação ao longo de } 6 \text { meses. (Psicoeducação) }\end{array}$ & $\begin{array}{c}\text { Menor depressão e estresse além de estratégias melhores } \\
\text { de saúde e enfrentamento. }\end{array}$ \\
\hline Oncologia (21) & $\begin{array}{l}\text { Foi avaliado as necessidades do cuidador, estabelecimento } \\
\text { de um plano de cuidados e fornecimento de apoio ao cuida- } \\
\text { dor. (Suporte prático ao cuidar e avaliação de necessidades) }\end{array}$ & $\begin{array}{l}\text { Houve mudanças no sofrimento. Nenhuma diferença signi- } \\
\text { ficativa foi encontrada entre a intervenção de duas visitas e } \\
\text { o grupo controle. }\end{array}$ \\
\hline Oncologia (22) & $\begin{array}{l}\text { Visitas domiciliares por vídeo e chamadas telefônicas. } \\
\text { (Suporte prático ao cuidar, avaliação de necessidades e } \\
\text { psicoeducação) }\end{array}$ & $\begin{array}{c}\text { Maior qualidade de vida, alta satisfação com o suporte } \\
\text { social, proximidade com o paciente, auto eficácia no } \\
\text { autocuidado, recompensas por cuidar e menor estresse e } \\
\text { depressão. }\end{array}$ \\
\hline Oncologia ${ }^{(23)}$ & $\begin{array}{l}\text { Um livreto com informações para cuidados familiares foi } \\
\text { distribuído (Suporte prático ao cuidar) }\end{array}$ & $\begin{array}{l}\text { Melhora na positividade em relação ao cuidado e maior } \\
\text { competência da sua função. }\end{array}$ \\
\hline Oncologia (24) & $\begin{array}{c}\text { Grupo psicopedagógico liderado por enfermeiros, em três } \\
\text { sessões. (Psicoeducação) }\end{array}$ & Melhoria na preparação e competência para cuidar. \\
\hline${ }^{*} A V E^{(25)}$ & $\begin{array}{c}\text { Fornecimento de orientações e avaliação das competên- } \\
\text { cias práticas. (Suporte prático ao cuidar e avaliação de } \\
\text { necessidades) }\end{array}$ & $\begin{array}{c}\text { Foi constatado menor sobrecarga de trabalho no grupo } \\
\text { experimental em detrimento do controle. }\end{array}$ \\
\hline${ }^{*} \mathrm{AVE}^{(26)}$ & $\begin{array}{l}\text { Acompanhamento sistemático (Suporte prático ao cuidar e } \\
\text { avaliação de necessidades) }\end{array}$ & $-{ }^{* *}$ \\
\hline
\end{tabular}

\section{DISCUSSÃO}

Esta revisão explorou na literatura científica as principais intervenções de enfermagem à cuidadores familiares e os principais efeitos das mesmas. Apenas treze artigos foram analisados, mediante os critérios de elegibilidade, o que corrobora com pesquisadores que já afirmavam sobre a escassez de pesquisas sobre tais intervenções ${ }^{(28)}$.

As intervenções de enfermagem analisadas neste estudo estavam voltadas apenas a cuidadores familiares de pacientes oncológicos em cuidados paliativos e àqueles vitimados por acidente vascular encefálico (AVE). Nove estudos foram com cuidadores de pacientes com câncer e isto se justifica devido ao aumento expressivo nos casos de pacientes oncológicos em cuidados paliativos nos últimos anos, o que demanda dos cuidadores mais tempo por dia em tarefas de cuidado ${ }^{(29)}$.

Cinco estudos avaliaram intervenções o oue demanda de enfermagem a cuidadores familiares dos cuidadores mais tempo por dia em tarefas de cuidado. de pacientes vítimas de AVE. Este por sua vez é uma das principais causas de deficiências graves que afeta cerca de 32,2 milhões de pessoas em todo o mundo, gerando uma recuperação longa e fazendo com que a família assuma na maior parte das vezes as tarefas de cuidado ${ }^{(30)}$.

Em relação aos componentes de intervenção identificados nos estudos analisados nos resultados, estes se baseiaram em três eixos principais: suporte prático 
ao cuidar, avaliação das necessidades e psicoeducação. O primeiro eixo foi o componente mais comum nas intervenções e teve como objetivo primário capacitar os cuidadores familiares acerca dos cuidados básicos realizados com os pacientes domiciliares.

O fato de 9 dos 13 estudos analisados realizarem intervenções no processo do cuidar é sustentado por pesquisas que demonstraram que os cuidadores familiares sentem-se despreparados em relação ao suporte prático que prestam a seus pacientes $^{(31,32)}$. Esse despreparo ocorre essencialmente no reconhecimento e monitorização de sintomas e na identificação de um momento adequado para entrar em contato com um profissional de saúde ${ }^{(32)}$.

A necessidade de melhorar as habilidades dos cuidadores familiares também foi um componente de intervenção de enfermagem das publicações da amostra. Nesse sentido, alguns estudos de revisão sistemática apontam para uma série de situações que envolvem a necessidade dos cuidadores familiares em receber intervenções de enfermagem, especialmente daqueles que cuidam de pacientes em cuidados paliativos $^{(32,33)}$. Estas necessidades se baseiam nas seguintes prerrogativas expostas por cuidadores familiares: ter suporte de saúde mental; obter informações sobre equipamentos médicos disponíveis no domicílio, como lidar com uma emergência e ter informações precisas e objetivas sobre como obter ajuda de um profissional de saúde ${ }^{(33)}$.

O componente psicoeducacional também foi alvo dos enfermeiros na realização das intervenções. Dos cinco estudos que realizaram esta ação, três tinham como objetivo realizar apenas a intervenção em psicoeducação. Estas intervenções são relatadas como benéficas para os cuidadores famíliares, de modo que alguns estudos encontraram efeitos positivos na preparação, competência, recompensas e nas necessidades dos cuidadores familiares ${ }^{(28,34)}$.

No tocante aos resultados das intervenções de enfermagem aos cuidadores familiares foi possível verificar que hou-
“

ve um efeito positivo e bastante significativo em todos os estudos analisados. Houve melhorias no desenvolvimento de habilidades na resolução de problemas e diminuição nos índices de depressão, sofrimento e estresse, o que demonstra que intervenções realizadas por enfermeiros tem efeitos tanto no componente do processo do cuidar quanto na saúde mental dos cuidadores familiares.

Porém poucos estudos tiveram resultados baseados na saúde mental e isto pode ser devido à alta taxa de cuidadores deprimidos. Outra justificativa é que cuidar de um membro da família pode ser inevitavelmente estressante e provocar sentimentos de ansiedade e depressão apesar da meIhor intervenção possível ${ }^{(35)}$.

De modo que os cuidadores familiares cuidam de seus entes queridos em suas casas por meses ou anos e, durante o curso da doença, forneçam cuidados e apoio extensivos que pode ser física, emocional e socialmente exigente, intervenções de enfermagem a estes indivíduos devem ser mais incentivadas a fim de garantir que eles prestem cuidados de maneira mais segura e que possam se sentir mais confiantes e preparados.

A principal limitação deste de estudo de revisão se refere as poucas publicações incluídas, sugerindo-se que este tema seja pouco abordado no âmbito da enfermagem. Porém, apesar desta limitação os estudos analisados apresentam delineamento metodológico consistente e de elevado nível de evidência, contribuindo de maneira significativa para as práticas de enfermagem baseada em evidências científicas.

\section{CONCLUSÃO}

Embora existam poucos artigos que abordem intervenções de enfermagem a cuidadores familiares, esta revisão analisou que as publicações já existentes possuem elevado rigor metodológico com intervenções claras e concisas.

Verificou-se as principais intervenções de enfermagem no âmbito do prepa- 
ro de cuidadores familiares se basearam em três eixos: suporte prático ao cuidar, avaliação das necessidades do cuidador e a psicoeducação. As intervenções realizadas pelos enfermeiros tiveram efeitos positivos entre os cuidadores familiares, sugerindo que a enfermagem têm função importante no apoio a estes indivíduos.

Desta forma as intervenções de enfermagem a cuidadores familiares po- dem beneficiar não apenas estes profissionais mas também os pacientes que recebem o cuidado, visto que os mesmos estarão mais capacitados e preparados para a sua realização.

\section{Referências}

1. Instituto Brasileiro de Geografia e Estatística - IBGE. Expectativa de vida dos brasileiros aumenta para 76,3 anos em 2018. Agência IBGE notícias; 2019. Disponível em: https://agenciadenoticias.ibge.gov.br/agencia-noticias/2012-agencia-de noticias/noticias/26103-expectativa-de-vida-dos-brasileiros-aumenta-para-76-3-anos-em-2018.

2. Sociedade Brasileira de Geriatria e Gerontologia - SBGG. ATIVIDADES de vida diária - o que são? SBGG; 2015. Disponível em: http://www.sbgg-sp.com. br/pub/atividades-da-vida-diaria-o-que-sao/.

3. Araújo 0, Lage I, Cabrita J, Teixeira L. Intervention in informal caregivers who take care of older people after stroke (InCARE): study protocol for a randomized trial. J Adv Nurs. 2015;71(10):2435-43.

4. Brasil. Ministério da Saúde. Guia Prático do Cuidador. Ministério da Saúde; 2008. Disponível em: http://bvsms.saude.gov.br/bvs/publicacoes/guia_pratico_cuidador.pdf.

5. Diniz ASS, Lima RA, Silva BRS. Sobrecarga do cuidador de idoso: uma revisão integrativa. Rev Pesq. Saúde. 2017; 18(3): 184-8. Disponível em: http://www.periodicoseletronicos.ufma.br/index.php/revistahuufma/article/ view/8598/5968.

6. Brasil. Ministério da Saúde. Portaria $n^{\circ}$ 963, de 27 de maio de 2013. Redefine a Atenção Domiciliar no âmbito do Sistema Único de Saúde (SUS). Ministério da Saúde; 2013. Disponível em: http://bvsms.saude.gov.br/bvs/ saudelegis/gm/2013/prt0963_27_05_2013.html.

7. Fernandes CS, Angelo M. Family caregivers: what do they need? An integrative review. Rev Esc Enferm USP. 2016; 50(4):672-8.

8. Day CB, Bierhals CCBK, Santos NOD, Mocellin D, Predebon ML, Dal Pizzol FLF, et al. Nursing home care educational intervention for family caregivers of older adults post stroke (SHARE): study protocol for a randomised trial. Trials. 2018; 19(1):96.

9. Silva RCA, Monteiro GL, Santos AG. Nurses' role in the education of caregivers of patients with stroke. Rev de Atenção à Saúde. 2015;13(45):114-20.

10. Gil AC. Como elaborar projetos de pesquisa: 6. Ed. São Paulo: Atlas; 2017. 11. Pompeo DA, Rossi LA, Galvão CM. Revisão integrativa: Etapa inicial do processo de validação de diagnóstico de enfermagem. Acta Paul Enferm. 2009; 22(4):434-8.

12. Souza MAO, Souza NR, Melo JTS, Xavier MACA, Almeida GL, Santos ICRV. Odor evaluation scales for odor in neoplastic wounds: an integrative review. Rev Bras Enferm. 2018; 71(5):2552-60.

13. Stillwell S, Melnyk BM, Fineout-Overholt E, Williamson K. Evidence- based practice: step by step. Am J Nurs. 2010;110(5):41-7.

14. Brasil. Ministério da Saúde. Lei no 9.610, de 19 de fevereiro de 1998, que altera, atualiza e consolida a legislação sobre direitos autorais e dá outras providências. Diário Oficial da União. Brasília: Ministério da Saúde; 1998.

15. Grant JS, Elliott TR, Weaver M, Bartolucci AA, Giger JN. Telephone intervention with family caregivers of stroke survivors after rehabilitation. Stroke. 2002; 33(8):2060-5.

16. Hudson PL, Aranda S, Hayman-White K. A psycho-educational interven tion for family caregivers of patients receiving palliative care: a randomized controlled trial. J. Pain Symptom Manage. 2005; 30(4): 329-341.

17. Hudson $P$, Quinn $K$, Kristjanson L. Evaluation of a psycho-educational group programme for family caregivers in home-based palliative care. Palliat. Med. 2008; 22(3): 270-80.

18. Hudson P, Trauer T, Kelly B, O'Connor M, Thomas K, Summers M, et al. Reducing the psychological distress of family caregivers of home-based palliative care patients: short-term effects from a randomised controlled trial. PsychoOncology. 2013; 22(9): 1987-93.
19. Ostwald SK, Godwin KM, Cron SG, Kelley CP, Hersch G, Davis S. Home-based Psychoeducational and Mailed Information Programs for Stroke-Caregiving Dyads Post-Discharge: A Randomized Trial. Disabil Rehabil. 2013; 36(1):55-62. 20. Aoun SM, Grande G, Howting D, Deas K, Toye C, Troeung L, et al. The impact of the carer support needs assessment tool (CSNAT) in community palliative care using a stepped wedge cluster trial. PLoS One. 2015; 10(4): e0123012. 21. Hudson $P$, Trauer T, Kelly B, O'Connor M, Thomas $K$, Zordan R, et al. Reducing the psychological distress of family caregivers of home based palliative care patients: Longer term effects from a randomised controlled trial. PsychoOncology. 2015; 24(1): 19-24.

22. Leow M, Chan S, Chan MF. A pilot randomized, controlled trial of the effectiveness of a psychoeducational intervention on family caregivers of patients with advanced cancer. Oncol. Nurs. Forum. 2015; 42(2): E63-E72.

23. Luker K, Cooke M, Dunn L, Lloyd-Williams M, Pilling M, Todd C. Development and evaluation of an intervention to support family caregivers of people with cancer to provide home-based care at the end of life: a feasibility study. Eur. J. Oncol. Nurs. 2015; 19(2): 154-61.

24. Holm M, Årestedt K, Carlander I, Fürst CJ, Wengström Y, Öhlen J, et al. Short-term and long-term effects of a psycho-educational group intervention for family caregivers in palliative home care - results from a randomized control trial. PsychoOncology. 2016; 25(7): 795-802.

25. Araújo 0, Lage I, Cabrita J, Teixeira L. Eficácia do programa InCARE na sobrecarga dos cuidadores informais de pessoas idosas após um AVC. Rev. port. enferm. saúde mental. 2016; 3: 9-13.

26. Day CB, Bierhals CCBK, Santos NO, Mocellin D, Predebon ML, Dal Pizzol FLF. Nursing Home Care Educational Intervention for Family Caregivers of Older Adults Post Stroke (SHARE): Study Protocol for a Randomised Trial. Trials. 2018; 19(1):96.

27. Cheng HY, Chair SY, Chau JPC. Effectiveness of a Strength-Oriented Psychoeducation on Caregiving Competence, Problem-Solving Abilities, Psychosocial Outcomes and Physical Health Among Family Caregiver of Stroke Survivors: A Randomised Controlled Trial. Int J Nurs Stud. 2018; 87: 84-93.

28. Hudson PL, Remedios C, Thomas K. A systematic review of psychosocial interventions for family carers of palliative care patients. BMC Palliat. Care. 2010; 9(1): 17.

29. Streck BP, Wardell DW, LoBiondo-Wood G, Beauchamp JES. Interdependence of Physical and Psychological Morbidity Among Patients With Cancer and Family Caregivers: Review of the Literature.

Psychooncology. 2020.

30. Feigin VL, Norrving B, Mensah GA. Global burden of stroke. Circ. Res. 2017; 120: 439-88.

31. Candy $B$, Jones $L$, Drake $R$, Leurent $B$, King $M$. Interventions for supporting informal caregivers of patients in the terminal phase of a disease. Cochrane Database Syst Rev. 2011; 6.

32. Applebaum AJ, Breitbart W. Care for the cancer caregiver: a systematic review. Palliat Support Care. 2011; 11(3):231.

33. Ventura AD, Burney S, Brooker J, Fletcher J, Ricciardelli L. Home-based palliative care: a systematic literature review of the self-reported unmet needs of patients and carers. Palliat Med. 2014; 28(5):391.

34. Hartmann M, Bäzner E, Wild B, Eisler I, Herzog W. Effects of interventions involving the family in the treatment of adult patients with chronic physical diseases: a meta-analysis. Psychother. Psychosom. 2010; 79(3): 136-48. 35. Becquéa YN, Rietjens JAC, Driela AGV, Heideb AVD, Witkampa E. Nursing interventions to support family caregivers in end-of-life care at home: A systematic narrative review. Int J Nurs Stud. 2019; 97: 28-39. 